\author{
A. Schibler \\ T. M. T. Pham \\ K. R. Dunster \\ K. Foster \\ A. Barlow \\ K. Gibbons \\ J. L. Hough
}

\section{Reduced intubation rates for infants after introduction of high-flow nasal prong oxygen delivery}

Received: 26 July 2010

Accepted: 17 January 2011

Published online: 3 March 2011

(C) Copyright jointly held by Springer and ESICM 2011
Abstract Purpose: To describe the change in ventilatory practice in a tertiary paediatric intensive care unit (PICU) in the 5-year period after the introduction of high-flow nasal prong (HFNP) therapy in infants $<24$ months of age. Additionally, to identify the patient subgroups on HFNP requiring escalation of therapy to either other non-invasive or invasive ventilation, and to identify any adverse events associated with HFNP therapy. Methods: The study was a retrospective chart review of infants $<24$ months of age admitted to our PICU for HFNP therapy. Data was also extracted from both the local database and the Australian New Zealand paediatric intensive care (ANZPIC) registry for all infants admitted with bronchiolitis. Results: Between January 2005 and December 2009, a total of 298 infants $<24$ months of age received HFNP therapy. Overall, 36 infants $(12 \%)$ required escalation to invasive ventilation. In the subgroup with a primary diagnosis of viral bronchiolitis $(n=167,56 \%)$, only $6(4 \%)$ required escalation to invasive ventilation. The rate of intubation in infants with viral bronchiolitis reduced from $37 \%$ to $7 \%$ over the observation period corresponding with an increase in the use of HFNP therapy. No adverse events were identified with the use of HFNP therapy. Conclusion: HFNP therapy has dramatically changed ventilatory practice in infants $<24$ months of age in our institution, and appears to reduce the need for intubation in infants with viral bronchiolitis.

Keywords High-flow nasal cannula . Oxygen delivery · Infant
K. Gibbons · J. L. Hough

Mater Medical Research Institute,

Mater Health Services, South Brisbane, QLD, Australia dedicated CPAP driver $[4,5]$. Recently high-flow nasal prong (HFNP) therapy has been introduced to provide respiratory support in preterm and term infants [6-8]. HFNP therapy has many possible advantages over other forms of oxygen therapy: the inspired gas mixture can be heated and humidified to reduce damage to the upper airway mucosa; the inspired oxygen concentration can be titrated to the patient's need; anecdotally, it is better tolerated by the patient; and potentially, CPAP can be delivered [9-12]. Studies in neonates have shown that the amount of CPAP delivered by HFNP depends on the flow and generated by a water column (bubble CPAP) or a
Respiratory distress and hypoxaemia in infants are treated with various forms of non-invasive respiratory therapy . In addition to oxygen therapy, continuous posi breathing and improve functional residual capacity, regional atelectasis of the lung is a common feature in infants breathing near their closing volume $[2,3]$. CPAP
can be delivered via nasopharyngeal tube or face mask 
(relative to the size of the patient) and on the leak around the nasal cannula [13]. Most studies of HFNP therapy have been performed in neonates, and little clinical experience is reported in older children [6].

HFNP therapy was introduced in our paediatric intensive care unit (PICU) in 2005 and a subsequent significant change has been observed in the care of infants with respiratory distress with a suspected reduction in invasive ventilation. This retrospective analysis aimed to describe: our 5-year institutional experience of HFNP therapy in infants $<24$ months of age; the subgroups of patients requiring HFNP; the need for escalation of respiratory support from HFNP to other forms of non-invasive or invasive ventilation; the incidence of complications associated with HFNP therapy; our ventilatory practice in comparison with the subgroup of infants with bronchiolitis in all other PICUs in Australia and New Zealand.

\section{Methods}

Study design

The study was a retrospective analysis of all infants admitted to the PICU and treated with HFNP therapy between 2005 and 2009. Demographic and physiological real-time data were extracted from the unit's clinical information system (CIS). Informed consent from parents or guardians was waived by the local ethics committee.

\section{Setting}

The PICU is a 19-bed tertiary mixed surgical/cardiac/medical unit with approximately 1,100 admissions per year.

Definition of patient disease groups

The database was queried for all infants $<24$ months of age who were treated with HFNP therapy within their first $24 \mathrm{~h}$ of admission to the PICU. These infants were allocated to six disease groups using the Australian New Zealand Paediatric Intensive Care (ANZPIC) registry coding criteria [14]: clinically defined viral bronchiolitis with or without a positive test for respiratory syncytial virus (RSV), adenovirus, metapneumovirus or influenza $(\mathrm{BRONCH})$; lung disease without peripheral airway obstruction (LD); upper airway obstruction (UAO); neuromuscular conditions (NM); cardiac conditions (CARDIAC); and other (OTHER). Within the BRONCH disease group, preexisting risk factors such as prematurity, or underlying cardiac or neurological disorders were also identified. Criteria for admission to the PICU for respiratory distress is an oxygen requirement of more than
$21 /$ min and the need for respiratory support additional to supplemental oxygen.

Definition of respiratory support subgroups

Infants within each disease group were then divided into respiratory support subgroups, as follows: HFNP only (HF), HFNP followed by other non-invasive ventilation $(\mathrm{HF}+\mathrm{N})$, HFNP followed by other non-invasive, followed by invasive ventilation $(\mathrm{HF}+\mathrm{N}+\mathrm{I})$, or HFNP followed by invasive ventilation $(\mathrm{HF}+\mathrm{I})$.

\section{HFNP system}

A humidified high-flow system was used with a low-resistance paediatric binasal cannula (BC3780 and RT329; Fisher $\&$ Paykel Healthcare, Auckland, New Zealand). The inspired oxygen concentration was titrated to achieve pulse oximeter oxygen saturations $\left(\mathrm{SpO}_{2}\right)$ of $>94 \%$. The flow rate used was generally set at $8 \mathrm{l} / \mathrm{min}$ at the beginning of the HFNP treatment and then weaned at the discretion of the attending consultant, most commonly down to 6 or $41 / \mathrm{min}$. Failure of HFNP therapy was defined as the need for escalation of therapy to either non-invasive ventilation with a face mask, or invasive ventilation with an endotracheal tube delivered by the ventilator (Evita XL; Draeger, Lubeck, Germany). Discontinuation of HFNP therapy was based on reduced oxygen requirement (generally inspired oxygen fraction $<0.4$ ), and clinical improvement in the work of breathing, respiratory rate (RR) and heart rate (HR).

\section{Patient parameters}

Body weight, age at admission, length of stay (LOS), and paediatric index of mortality risk of death (PIM2 ROD) score were recorded [15]. Additionally, LOS and intubation rate of all infants with viral bronchiolitis admitted to all PICUs in Australia and New Zealand were extracted from the most recent ANZPIC data registry and compared to our dataset [16].

\section{Continuous physiological variables}

Ventilatory parameters and physiological variables, such as $\mathrm{HR}, \mathrm{RR}, \mathrm{SpO}_{2}$, inspired oxygen fraction $\left(\mathrm{FiO}_{2}\right.$, value when initially started on HFNP therapy) and $\mathrm{SpO}_{2} / \mathrm{FiO}_{2}$ ratio were downloaded every $30 \mathrm{~min}$. Data were extracted from $4 \mathrm{~h}$ prior to initiation of HFNP therapy and continued for $24 \mathrm{~h}$. All data were extracted from the unit's CIS (Critical Care Manager; PICIS, Wakefield, MA). Data were either automatically downloaded from monitors and validated or manually entered by the bedside nurse. 
Table 1 Disease groups and respiratory support mode

\begin{tabular}{lrrlrr}
\hline Group & \multicolumn{1}{l}{ HF } & HF $+\mathrm{N}$ & $\mathrm{HF}+\mathrm{N}+\mathrm{I}$ & $\mathrm{HF}+\mathrm{I}$ & \multicolumn{1}{c}{ All } \\
& & & & & $\mathrm{HF}$ \\
\hline BRONCH & $120(72 \%)$ & $41(25 \%)$ & $4(2 \%)$ & $2(2 \%)$ & 167 \\
LD & $55(76 \%)$ & $9(13 \%)$ & $4(6 \%)$ & $4(6 \%)$ & 72 \\
UAO & $6(75 \%)$ & $0(0 \%)$ & $1(13 \%)$ & $1(13 \%)$ & 8 \\
NM & $8(80 \%)$ & $1(10 \%)$ & $0(0 \%)$ & $1(10 \%)$ & 10 \\
CARDIAC & $11(46 \%)$ & $1(4 \%)$ & $0(0 \%)$ & $12(50 \%)$ & 24 \\
OTHER & $6(35 \%)$ & $4(24 \%)$ & $1(6 \%)$ & $6(35 \%)$ & 17 \\
Total & $206(69 \%)$ & $56(19 \%)$ & $10(9 \%)$ & $26(3 \%)$ & 298 \\
\hline
\end{tabular}

\section{Adverse outcomes}

A system for prospectively coding complications is included in the CIS. These fields and the clinical notes were screened for adverse events such as cardiac and respiratory arrest, pneumothorax, gastric distension and mucosal injury due to cannula position. The number of failed HFNP treatments in respect of the need for other non-invasive and invasive ventilation was recorded.

\section{Comparison with ANZPIC registry}

To investigate the change in ventilator practice over time in our unit, case records were extracted from the local ICU database for all children admitted during the study period with bronchiolitis. In addition, case records were extracted from the ANZPIC Registry [14] for patients with bronchiolitis admitted during 2008. Intubation rate and LOS in our unit in 2009 were compared to the ANZPIC Registry patients in 2008.

\section{Statistical analysis}

Study groups, including the ANZPIC dataset, were compared using Fisher's exact test for categorical variables and variables presented as percentages. Wilcoxon's ranksum test was used to compare admission parameters and LOS. Data are presented as medians and interquartile ranges unless otherwise stated. To determine whether there was an impact of underlying disease or time since initiation of treatment on the change in the physiological variables, a linear mixed model was used. $\mathrm{HR}, \mathrm{RR}, \mathrm{SpO}_{2}$ and $\mathrm{SpO}_{2} / \mathrm{FiO}_{2}$ ratio were used as dependent variables, patient group and HFNP therapy sequence as factors, and time as a covariate.

\section{Results}

Between January 2005 and December 2009 a total of 298 infants received HFNP therapy in our PICU. Table 1 presents the distribution by disease group and respiratory support mode. Overall, 56 (19\%) infants receiving HFNP therapy needed escalation to other non-invasive and 36 (12\%) to invasive ventilation. Of the infants with a primary diagnosis of viral bronchiolitis, only $6(4 \%)$ required escalation to invasive ventilation. There was a significantly greater incidence of invasive ventilation in the CARDIAC $(n=12,50 \%)$ and OTHER $(n=7,41 \%)$ groups compared with the BRONCH $(n=6,4 \%)$ and LD $(n=8,12 \%)$ groups $(p<0.05)$. Most of the cardiac infants needed intubation for a cardiac surgical procedure or cardiac failure.

Table 2 shows the admission parameters and LOS of infants with viral bronchiolitis only. Significant differences were found between the HF group and the infants requiring escalation to other non-invasive ventilation for PIM2 ROD score, admission $\mathrm{FiO}_{2}$ and LOS $(p<0.01)$. There were no differences found between the therapy groups in terms of age. Although PIM2 ROD score and LOS were higher in the $\mathrm{HF}+\mathrm{N}+\mathrm{I}$ and $\mathrm{HF}+\mathrm{I}$ groups compared to the HF group, statistical comparison was not applicable due to the low number of infants in each group.

Table 3 shows the increased use of HFNP therapy in our PICU for viral bronchiolitis over the study period. In comparison to Table 1, all patients intubated at admission were included. In 2005, 52 infants were admitted with only 7 infants receiving HFNP therapy, whereas in 2009, 44 of 67 infants with bronchiolitis were started on HFNP
Table 2 Admission parameters of infants with bronchiolitis (medians and interquartile ranges)

\begin{tabular}{llllll}
\hline Parameter & $\begin{array}{l}\mathrm{HF} \\
(n=120)\end{array}$ & $\begin{array}{l}\mathrm{HF}+\mathrm{N} \\
(n=41)\end{array}$ & $\begin{array}{l}\mathrm{HF}+\mathrm{N}+\mathrm{I} \\
(n=4)\end{array}$ & $\begin{array}{l}\mathrm{HF}+\mathrm{I} \\
(n=2)\end{array}$ & $\begin{array}{l}\text { All HF } \\
(n=167)\end{array}$ \\
\hline PIM2 ROD score (\%) & 0.17 & 0.70 & 0.72 & 1.49 & 0.21 \\
& $0.16-0.23^{\#}$ & $0.59-0.87^{\#}$ & $0.60-0.83$ & - & $0.16-0.59$ \\
$\mathrm{FiO}_{2}$ & 0.50 & 0.60 & 0.60 & 0.45 & 0.50 \\
& $0.40-0.60^{\$}$ & $0.50-0.70^{\$}$ & $0.55-0.70$ & - & $0.4-0.6$ \\
LOS (days) & 1.83 & 3.75 & 9.35 & 16.9 & 2.33 \\
& $1.44-2.75^{\&}$ & $3.0-6.0^{\&}$ & $6.81-12.81$ & - & $1.6-3.5$ \\
Age (months) & 2.75 & 3.11 & 5.87 & 4.4 & 2.98 \\
& $1.43-7.62$ & $1.31-7.87$ & $4.11-6.85$ & - & $1.4-7.9$ \\
Weight (kg) & 5.50 & 5.20 & 6.10 & 6.15 & 5.46 \\
& $3.97-7.95$ & $3.90-7.70$ & $5.35-6.83$ & - & $3.96-7.85$ \\
\hline$\#, \$, \& p<0.01$ & & & & &
\end{tabular}


Table 3 Infants with viral bronchiolitis listed by year

\begin{tabular}{llcc}
\hline Year & $\begin{array}{l}\text { Total } \\
\text { BRONCH }\end{array}$ & HF and $\mathrm{HF}+\mathrm{N}$ & Total intubated \\
\hline 2005 & 52 & $7(13 \%)$ & $19(37 \%)$ \\
2006 & 72 & $32(44 \%)$ & $21(29 \%)$ \\
2007 & 49 & $23(46 \%)$ & $15(31 \%)$ \\
2008 & 90 & $56(62 \%)$ & $12(13 \%)$ \\
2009 & 67 & $44(66 \%)$ & $5(7 \%)$ \\
Total & 330 & $161(49 \%)$ & $72(22 \%)$ \\
\hline
\end{tabular}

therapy and only $5(7 \%)$ required intubation and ventilation. The overall intubation rate in our unit dropped from $37 \%$ in 2005 to $7 \%$ in 2009 whereas, in 2008, the ANZPIC data registry reported an overall intubation rate of $28 \%$.

The median LOS in the 2008 ANZPIC registry for bronchiolitic infants was 2.42 compared to 2.33 in our study $(p=\mathrm{ns})$.

Table 4 shows admission parameters for infants with LD. There were significant differences between the HF and $\mathrm{HF}+\mathrm{N}$ groups with lower PIM2 ROD score and $\mathrm{FiO}_{2}$ and shorter LOS $(p<0.001)$ in the HF group. Statistical comparison for the $\mathrm{HF}+\mathrm{N}$ and $\mathrm{HF}+\mathrm{N}+\mathrm{I}$ groups was not applicable due to low numbers.

Analysis of continuous physiological variables

In all infants there was a significant reduction in RR and HR after initiation of HFNP therapy $(p<0.001)$. There was a significant interaction between disease group and HR and RR as well as between HFNP therapy and HR and RR (mixed linear model, $p<0.001$ ). The patients with viral bronchiolitis had the greatest change in HR and RR after initiation of HFNP therapy (Figs. 1 and 2). After 90 min the mean RR and mean HR had both decreased by more than $20 \%$ of the baseline (mean decrease in RR was 7.0 breaths/min, 95\% CI 4.2-9.8, $p<0.05$, and mean decrease in HR was 13 beats/min, 95\% CI 9.25-16.75, $p<0.05)$ in the HF group whereas the in $\mathrm{HF}+\mathrm{N}$ group similar rapid decreases in RR and HR could not be demonstrated (mean decrease in RR was 5.0 breaths $/ \mathrm{min}$, 95\% CI 0-10, and mean decrease in HR was 6 beats $/ \mathrm{min}$,
95\% CI 0.6-11.4). There was no significant interaction between HFNP therapy and $\mathrm{SpO}_{2}$ or $\mathrm{SpO}_{2} / \mathrm{FiO}$ ratio.

\section{Adverse effects}

There were two in-hospital respiratory arrests and one inhospital cardiac arrest identified in the database, all three of which occurred before admission to the PICU and before the start of HFNP. No pneumothorax, gastric or abdominal distension or mucosal injuries were identified.

\section{Discussion}

In this retrospective analysis we showed that since the introduction of HFNP therapy in our PICU, the need for intubation and mechanical ventilation in infants with viral bronchiolitis decreased significantly over the 5-year period, from $37 \%$ in 2005 to $7 \%$ in 2009 . A similar reduction in intubation rate for bronchiolitic patients has also been reported in a retrospective study by McKiernan et al. [6]. They reported a reduction from $23 \%$ to $9 \%$, but did not report whether non-invasive ventilation with a face mask was used in their unit. This reduction reported in our unit is unlikely to be explained by an overall improved standard of care across time, as the ANZPIC registry data for 2008 still reported a comparatively higher ventilation rate for infants with bronchiolitis in a PICU [16]. As with any retrospective analysis, it is always difficult to demonstrate a cause and effect relationship, but there are some important findings in our retrospective analysis that may have been related to the reduced intubation rate.

The most common reason for non-elective admission to a PICU in Australia is viral bronchiolitis which imposes a significant financial burden on the hospital [16]. In our retrospective analysis, infants with viral bronchiolitis comprised the largest proportion of infants receiving HFNP therapy, followed by infants with lung disease. Similar to respiratory care of the preterm infant in the NICU [8], there has been an increasing trend toward the use of non-invasive ventilation in the PICU in order to reduce the risks associated with invasive ventilation. In our 5-year observation period, the proportion of infants
Table 4 Admission parameters for infants with lung disease (medians and interquartile ranges)

\begin{tabular}{llllll}
\hline Parameter & $\begin{array}{l}\mathrm{HF} \\
(n=55)\end{array}$ & $\begin{array}{l}\mathrm{HF}+\mathrm{N} \\
(n=9)\end{array}$ & $\begin{array}{l}\mathrm{HF}+\mathrm{N}+\mathrm{I} \\
(n=4)\end{array}$ & $\begin{array}{l}\mathrm{HF}+\mathrm{I} \\
(n=4)\end{array}$ & $\begin{array}{l}\text { All HF } \\
(n=72)\end{array}$ \\
\hline PIM2 ROD score (\%) & 0.83 & 3.61 & 3.23 & 1.77 & 0.91 \\
& $0.39-1.06^{\#}$ & $1.14-4.42^{\#}$ & $2.41-7.34$ & $0.61-6.58$ & $0.75-1.22$ \\
$\mathrm{FiO}_{2}$ & 0.5 & 0.6 & 0.58 & 0.55 & 0.5 \\
& $0.4-0.6^{\$}$ & $0.6-0.8^{\$}$ & $0.51-0.60$ & $0.50-0.63$ & $0.4-0.6$ \\
LOS (days) & 1.42 & 7.83 & 25.56 & 11.46 & 1.83 \\
& $0.88-2.69^{\&}$ & $5.42-16.46^{\&}$ & $20.69-42.02$ & $9.68-14.88$ & $0.96-4.31$ \\
\hline$\#, \$ p<0.01$ & & & & &
\end{tabular}




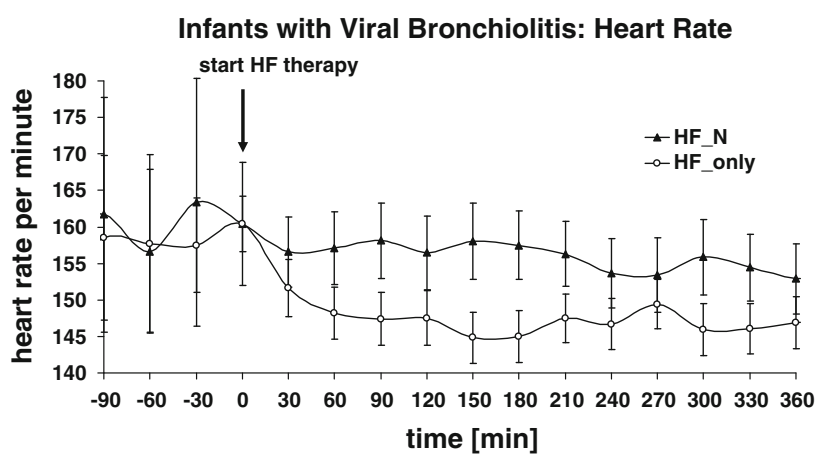

Fig. 1 HR in infants with viral bronchiolitis $90 \mathrm{~min}$ before and $6 \mathrm{~h}$ after the start of HFNP therapy. HR decreased significantly in the HF group (linear mixed model, $p<0.001$ ). Successfully treated infants in the HF group showed a significantly lower HR 90 min after the start of HFNP therapy than those in the HF $+\mathrm{N}$ group $(p<0.05)$. The data are presented as means and 95\% CI

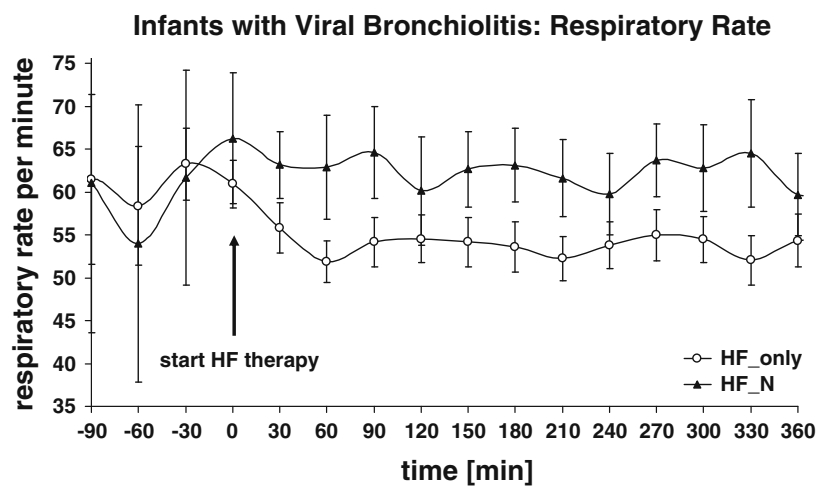

Fig. 2 RR of infants with viral bronchiolitis $90 \mathrm{~min}$ before and $6 \mathrm{~h}$ after the start of HFNP therapy. RR decreased significantly in the HF group (linear mixed model, $p<0.001$ ). Successfully treated infants in the HF group showed a significantly lower RR 90 min after start of HFNP therapy than those the $\mathrm{HF}+\mathrm{N}$ group $(p<0.05)$. The data are presented as means and 95\% CI

with viral bronchiolitis treated with HFNP increased from $13 \%$ to $66 \%$, while those requiring intubation decreased proportionately. The admission criteria did not change during this observational study. Our standard admission practice was that infants with respiratory distress and an increased oxygen requirement of $>21 / \mathrm{min}$ were reviewed by a PICU consultant or senior registrar either in the emergency department, paediatric ward or during retrieval from a referring hospital. Infants were only admitted to the PICU if respiratory support additional to supplemental oxygen was considered necessary.

Escalation of therapy to other non-invasive ventilation occurred in one-quarter of infants with bronchiolitis within the first $24 \mathrm{~h}$ of admission. The mean HR and mean RR discriminated between responders and nonresponders to HFNP therapy. Responders showed a $20 \%$ decrease in RR and HR within 90 min of the start of HFNP therapy, whereas non-responders showed little change in RR and HR. Infants who required escalation of treatment to other non-invasive ventilation had a higher PIM2 ROD score and $\mathrm{FiO}_{2}$ when HFNP therapy was started on admission.

Did the introduction of HFNP lead to longer LOS? The median LOS for all infants with bronchiolitis in our unit was no different from that reported in the ANZPIC registry data for 2008 [16]. With the increased experience, indications for HFNP were broadened and HFNP therapy was initiated in infants with causes of respiratory distress other than viral bronchiolitis. Infants with LD who needed escalation to other non-invasive ventilation were generally sicker on admission, demonstrating a higher PIM2 ROD score and $\mathrm{FiO}_{2}$ and had a longer LOS. In infants with cardiac disease the intubation rate was $50 \%$ within the first $24 \mathrm{~h}$ of admission suggesting that HFNP therapy was not as effective in this population of infants. This was due to the fact that these infants were intubated for a cardiac procedure or severe cardiac failure.

This study was limited to a single institution without a control group, and clinical practice changed over the study period. A multicentre randomized controlled trial comparing HFNP therapy with standard care is needed to assess and prove the efficacy of HFNP therapy.

In conclusion, HFNP therapy provided efficient respiratory support and oxygen delivery in infants with respiratory distress in our PICU, and its introduction coincided with a significant reduction in the need for intubation of infants with viral bronchiolitis. Further research is required to establish safety and efficacy of HFNP definitively.

Acknowledgment We are grateful to the Preston James Fund and Golden Casket for support of this study.

\section{References}

1. Frey B, Shann F (2003) Oxygen administration in infants. Arch Dis Child 88:F84-F88

2. Reid LM (1984) Lung growth in health and disease. Br J Dis Chest 78:113-134
3. Thorsteinsson A, Werner O, Jonmarker C, Larsson A (2002) Airway closure in anesthetized infants and children: influence of inspiratory pressures and volumes. Acta Anaesthesiol Scand 46:529-536
4. Klein M, Reynolds LG (1986) Relief of sleep-related oropharyngeal airway obstruction by continuous insufflation of the pharynx. Lancet 1:935-939 
5. Frey B, McQuillan PJ, Shann F, Freezer N (2001) Nasopharyngeal oxygen therapy produces positive endexpiratory pressure in infants. Eur $\mathbf{J}$ Pediatr 160:556-560

6. McKiernan C, Chua LC, Visintainer PF, Allen H (2010) High flow nasal cannulae therapy in infants with bronchiolitis. J Pediatr 156:634-638

7. Campbell DM, Shah PS, Shah V, Kelly EN (2006) Nasal continuous positive airway pressure from high flow cannula versus Infant Flow for preterm infants. J Perinatol 26:546-549

8. Shoemaker MT, Pierce MR, Yoder BA, DiGeronimo RJ (2007) High flow nasal cannula versus nasal CPAP for neonatal respiratory disease: a retrospective study. J Perinatol 27:85-91
9. Dysart K, Miller TL, Wolfson MR, Shaffer TH (2009) Research in high flow therapy: mechanisms of action. Respir Med 103:1400-1405

10. Spence KL, Murphy D, Kilian C, McGonigle R, Kilani RA (2007) Highflow nasal cannula as a device to provide continuous positive airway pressure in infants. J Perinatol 27:772-775

11. Wilkinson DJ, Andersen CC, Smith K, Holberton J (2008) Pharyngeal pressure with high-flow nasal cannulae in premature infants. J Perinatol 28:42-47

12. Spentzas T, Minarik M, Patters AB, Vinson B, Stidham G (2009) Children with respiratory distress treated with high-flow nasal cannula. J Intensive Care Med 24:323-328

13. Kubicka ZJ, Limauro J, Darnall RA (2008) Heated, humidified high-flow nasal cannula therapy: yet another way to deliver continuous positive airway pressure? Pediatrics 121:82-88
14. Slater A, Shann F, McEniery J (2003) The ANZPIC registry diagnostic codes: a system for coding reasons for admitting children to intensive care. Intensive Care Med 29:271-277

15. Slater A, Shann F (2004) The suitability of the pediatric index of mortality (PIM), PIM2, the pediatric risk of mortality (PRISM), and PRISM III for monitoring the quality of pediatric intensive care in Australia and New Zealand. Pediatr Crit Care Med 5:447-454

16. Alexander J, Tregea S, Slater A (2008) Report of the Australian and New Zealand Paediatric Intensive Care Registry. Australian and New Zealand Intensive Care Society, Carlton South, Victoria 